\title{
Entretien avec Annie Salager ${ }^{1}$ John Stout \\ McMaster University (Hamilton, Ontario)
}

JS: Annie, vous venez de publier Terra nostra, ${ }^{2}$ qui est une sélection de poèmes tirés de plusieurs des recueils que vous avez publiés jusqu'ici. À lire Terra nostra, on se rend compte que vous êtes l'auteur d'une oeuvre magistrale, l'une des plus impressionnantes de notre temps, à mon avis. Comment se fait-il que vous ayez choisi la poésie comme moyen d'expression plutôt que la prose? Qu'est-ce qui vous attire à la poésie?

AS: Pourquoi la poésie? La prose, le roman, en un sens c'est presque le contraire du poème. Le poème, c'est pour aller chercher la racine des mots - la racine, le sens, le chant. C'est presque ne pas dire. C'est aller contre le langage usuel, la communication. C"est aller chercher le feu sous la parole, réinventer la parole dans l'être humain. Le désir était là en moi de le faire. Je ne l'ai pas voulu, choisi. Tandis que le roman - la prose - c'est tout autre chose. C'est plus technique. C'est l'histoire. C'est un mouvement de pensée très différent. Mais, bien sûr, plus on maîtrise l'un, plus on peut aller vers l'autre. Au fond, ce sont deux démarches d'esprit différentes, deux désirs différents. La prose veut couvrir le monde et la poésie dans le poème veut le dévoiler.

JS: Parmi vos premiers recueils de poésie, je suis surtout fasciné par celui que vous avez publié en 1973, La Femme-buisson. Qu'est-ce que cette image de "la femmebuisson" signifie dans votre oeuvre? Pourquoi l'avez-vous reprise plus tard, dans Figures du temps sur une eau courante et Terra nostra? 
AS: $\quad$ Il me semble que cette image du buisson a à voir avec le côté féminin de l'esprit.

JS: Pourquoi avez-vous choisi une image du végétal plutôt que, par exemple, le minéral?

AS: Moi qui aime la nature, crois posséder une sensibilité vraie à la nature, les volcans, par exemple ne me font pas vibrer. Je n'ai pas envie de monter sur un volcan. C'est comme ça. C'est plutôt l'eau et le végétal qui portent à l'onirisme pour moi.

JS: Avec l'eau et le végétal, en lisant vos poèmes, je pense à la fluidité, à la transformation. Le végétal, c'est quelque chose qui n'est pas statique, mais qui change constamment. C'est relié aussi au langage poétique. Le poète se penche sur la nature, comme sur le langage poétique, se demande où les deux peuvent se rejoindre. Je pense que cette image de la femme-buisson traduit très bien l'analogie qu'il peut y avoir entre les deux.

AS: $\quad$ Il doit y avoir un rapport entre écriture et nature, effectivement. Pour moi, ce que j'aime est du côté de la fluidité, en effet - ce que vous dites. Le végétal bouge et je vois que je l'ai mis souvent dans mes poèmes. Puis, ce que j'adore, c'est l'eau — nager, plonger. Cette fluidité est une immersion panthéiste.

JS: Est-ce que vous avez le même genre de rapport au langage qu'à l'eau et au végétal?

AS: Je crois qu'à l'époque où j'écrivais cela, à ces époques-là où, en gros, j'écrivais des textes sur la mer, je suivais plus la proposition de la nature et mon langage se faisait fluide par une espèce d'imitation. Il me semble que, peu à peu, je le vois maintenant, je n'éprouve plus le même besoin de le dire, tout en jouissant beaucoup de la nature. Une fois qu'une chose a été dite, je n'ai plus envie de répéter cela. L'écriture et la nature ont dû se disjoindre un peu avec le temps au profit d'une meilleure qualité de mon écriture, j'imagine.

JS: Dans la nature, telle que vous l'évoquez, je suis fasciné également par le rôle des saisons. J'ai l'impression, en vous lisant, que les signes produits par la nature, et que le poète déchiffre, semblent inspirer et guider votre démarche. Est-ce l'idée du passage du temps, tel qu'il s'exprime à travers la nature, qui expliquerait l'importance des saisons dans votre poésie?

AS: C'est-à-dire j'en vois une, saison, que j'aime particulièrement et c'est le printemps. Chaque printemps, effectivement, j'ai envie d'écrire des poèmes. Indéfiniment. Ça me reprend chaque année de montrer la merveille de la nature qui fleurit. (Je parle des autres saisons aussi; l'été, je le vis plutôt). Mais le rapport avec l'écriture se situe là, à ce départ printanier. Cela doit donner un oeil attentif, une temporalité. Puis, vous savez, il y a la 
tradition espagnole du temps qui passe. Je suis hispaniste, donc je suis censée avoir lu quelques livres! Voilà les "coplas a la muerte de mi padre" de Manrique, la peinture de Valdez-Leal. Tout ce sens de la vie qui passe, de la finitude, de la mort, qui a à voir avec le baroque que j'aime, m'a toujours beaucoup plu - mais pas de façon intellectuelle. Je ne le savais pas d'avance. J'ai constaté après qu'il y avait des rapports avec le baroque. La culture hispanique a dû alimenter ce goût.

JS: $\quad$ Parmi les éléments de la nature, on dirait que ce sont surtout la terre et la mer qui se trouvent à l'origine de votre créativité. Même les titres de vos recueils l'indiquent, comme Récit des terres à la mer et Terra nostra. Comment employez-vous ces éléments dans votre poésie?

AS: Comment je les emploie? Au fond, qu'est-ce que ça me fait, un beau paysage, la terre que je perçois maintenant de plus en plus - un peu comme les Indiens - presque physiquement? Je pense à la terre comme un élément dont on est une partie. Ma vision s'est affinée, avec le temps, sur la terre.

JS: C'est la terre, avec la mer, qui donne un sentiment de l'immensité dans vos textes.

AS: Oui. Et du rapport surtout: qu'on est un bout de notre terre. Je crois qu'il y a là une conscience nouvelle de la part de l'Occident, qui est en train d'émerger. Il est temps! Mais comment je l'emploie - pour répondre à votre question - ou la terre, ou la mer; ça m'a donné du plaisir, des émotions et un désir de les traduire.

JS: Avec la terre et la mer, dans votre poésie, tout un côté cosmique s'affirme. La terre est à la fois un élément concret qu'on peut toucher, qui est là devant nous, comme on peut plonger dans la mer, et un indice du spirituel. La terre et la mer peuvent donner un sens du spirituel. Je sais que le spirituel est très important pour vous comme inspiration poétique ou même comme raison d'être de la poésie, n'est-ce pas?

AS: $\quad$ Oui, c'est vrai. Finalement, la poésie est une ascèse pour moi, au sens spirituel, un exercice spirituel, un exercice d'élucidation. Je l'ai vu peu à peu. Ce que j'ai bien senti, c'est que j'allais vers un dépouillement. J'ai quitté ceci, cela, la situation aisée, bien des amours. J'ai beaucoup quitté vers un repli qui m'assomme mais qui s'est fait, et se fait, malgré moi. Au profit de quoi? De cet aléatoire, de ce désir terrible de dire, et dont on voit en même temps que cela intéresse de moins en moins l'humanité. Presque comme un religieux qui passerait sa vie dans un couvent [là, j'exagère..]. Il y a, pourtant de cela assurément.

JS: Et qui trouve la grâce? 
AS: Qui trouve la grâce, mais qui trouve aussi le doute. Donc l' à quoi bon ne cesse de me rendre parfois amère.

JS: Est-ce que cela est un obstacle à l'écriture ou est-ce que cela alimente l'écriture, ce conflit?

AS: Je crois que cela l'alimente. Je suis bien obligée à posteriori, vu tout le passé, de constater que ça l'a alimenté, que je n'ai cherché que ça: un silence. Parce que quand on est une femme - je me suis mariée, j'ai eu mes deux fils très jeune - c'est un très long chemin de découvrir la poésie. D'autant plus que j'avais des interdits en moi-même.

JS: $\quad$ Est-ce qu'en prenant la parole en tant que femme à cette époque-là vous aviez l'impression de faire une espèce de transgression culturelle? Est-ce que cela a été difficile? Ou est-ce que cela vous a donné un sentiment de liberté très particulier?

AS: Le sentiment de liberté, je l'ai maintenant. Je l'ai eu à divers moments. Mais non, tout a été obstacle. J'ai bien eu l'impression, par exemple, que quitter mon mari et m'en aller, sans savoir exactement...Il y avait des motifs qui m'étaient à demi inconnus. Bien sûr, on a toujours des raisons objectives. Mais je savais aussi par quoi j'étais poussée: par ce désir de chercher une voix en moi. C'était aller contre moi et contre les autres. Donc je peux dire que ce n'était pas du tout commode. Non. Mais en même temps, $j$ 'en connaissais l'enjeu aussi, tout en sentant que j'étais moins que rien. Je ne me prenais pas plus au sérieux que maintenant, mais quelque chose en moi en connaissait l'éventuelle valeur. Une aventure humaine comme une autre, enfin. Le risque de la liberté.

JS: Ce que vous dites là me fait penser à la gazelle de vos poèmes. Le contact entre le poète et les bêtes, les animaux, revient souvent chez vous. Il y a l'oiseau, le martinet, en particulier. Il y a aussi la gazelle, surtout dans les poèmes récents. Pourquoi avez-vous choisi ces animaux, ces bêtes? S'agit-il d'une figure du poète et de la poésie?

AS: Oui, effectivement. Il y a trois séries d'animaux. Il y a les martinets, puisque je les ai là, au-dessus de chez moi. Image de la liberté et de l'immersion dans le cosmos. C'est le pur plaisir d'être. C'est comme l'essence même de l'être, le martinet. La gazelle est venue après. La gazelle, c'est l'anti-troupeau.

JS: Le troupeau, c'est plutôt une image dévalorisante, une image négative de l'humanité dans votre poésie. C'est vivre sans penser, sans réfléchir. Suivre des directives sociales. Vous voyez la gazelle, alors, en opposition à cela.

AS: Oui, mais, vous voyez, la gazelle n'est pas Antigone. C'est autre chose encore. Le 
troupeau, effectivement est cette si lente évolution de l'humanité, notre violence, notre agressivité incroyable...

JS: Que vous évoquez dans Les Dieux manquent de tout.

AS: Oui. Je me souviens que quand j'écrivais ces textes, je voyais des troupeaux, comme des troupeaux de bisons, ceux que les blancs ont tués en arrivant sur le continent américain, ce qu'on fait partout, quoi. Je voyais cela. Et c'est nous. Je disais que la gazelle, c'est pas Antigone. Antigone, c'est plus intellectuel, c'est plus volontaire elle la liberté assumée tandis que la gazelle est, je dirais, au-delà. C'est l'élément spirituel, plutôt un élément surgi de quelque chose de mystique, dans une imagerie dont je ne sais pas pourquoi elle me vient ainsi de l'Orient.

JS: Dans plusieurs poèmes vous opposez la gazelle au chasseur, à l'homme en tant que chasseur. S'agit-il d'une position féministe?

AS: $\quad$ Pas féministe, mais sûrement féminine. De même, j'ai réalisé seulement au moment de la Guerre du Golfe que finalement, l'humanité, c'est une espèce d'histoire du viol des femmes. C'est pas tellement dit, mais j'aurais pu le penser pendant la Guerre d'Algérie. Au fond, il n'y pas si longtemps, qu'on dit dans les médias que les femmes ont été violées. Tout d'un coup, je me suis dit: "Mais ça s'est fait depuis que le monde est monde!" Même avant que j'écrive ces histoires de chasseurs - Dieu merci, l'inconscient est plus savant que nous! — je le savais déjà. D'ailleurs, je crois qu'on l'hérite. On sait des tas de choses qu'on ignore, aussi.

JS: Dans ce contexte, est-ce que la gazelle est un signe d'espoir? Par la gazelle, est-ce que vous valorisez ce qui va à l'encontre de ce viol historique du féminin?

AS: La gazelle, je la sens comme je voudrais que le monde soit, que nous soyons tous, des initiés, tous des bodhisattvas, tous des Christs. Utopie. La gazelle c'est (révérence gardée!) comme les images de St. Jean de la Croix. Par exemple, quand l'animal vient boire à la source au divin. (Je ne suis pas très sûre de croire en Dieu, mais je crois que le divin, nous l'inventons, il est le produit de l'humanité. Voilà pourquoi il est encore si peu de chose. C'est un peu aussi ce que disent les Bouddhistes, il me semble).

JS: Vous évoquez les Bouddhistes. Vous avez parlé du Tao avec moi hier. Je sais que l'Orient, la pensée orientale, vous ont vraiment influencée. Dans certains de vos poèmes, vous vous adressez au grand poète chinois $\mathrm{Li}$ Po: " $\mathrm{Li}$ Po, buvons ce verre ensemble./ Vous ne voudriez pas, je pense, me le refuser!" Est-ce que vous pouvez parler de cette parenté que vous ressentez avec la pensée et la culture orientale? 
AS: Pour préparer un voyage en Chine, j'ai fait du chinois pendant trois ans. Et pour voir comment elle fonctionnait, cette poésie chinoise, avec ses caractères. J'ai compris que chaque caractère était musical, rythmique.

JS: $\quad$ Et image en même temps.

AS: Image et polysémie. En même temps, je lisais en français de la littérature chinoise. Je trouve que ce qu'il y a de génial vraiment chez les Chinois, c'est le mélange taoïste et confucianiste. Le Tao - pour reprendre la discussion à propos de $\mathrm{Li} \mathrm{Po}$, entre autres affiche l'image d'une dure liberté. C'est magnifique. Tandis que Confucius, c'est se plier à la société. Pardon d'être aussi sommaire! Je trouve que la Chine, armée de ces deux philosophies est très bien armée. J'ai aimé ce sens de la liberté qu'il y a à l'époque des Tang. Et plus tard, grâce au Dalai Lama, j'ai, comme des millions et des millions d'Occidentaux maintenant, aimé ce que j'ai lu et ce qu'il nous dit du Bouddhisme, qui parle plus à notre sensibilité contemporaine, plus que nos religions révélées, qu'on ne peut pas vraiment prendre au pied de la lettre. Hélas, il y en a une qui prend tout actuellement au pied de la lettre et ça fait des ravages! Périodiquement, on dit que tout vient d'Orient. Effectivement, l'Occident, à chaque fois, y a repris source. Je l'ai étudié un peu, pour le douzième siècle. Par les Arabes et les Juifs nous sont arrivées les cultures de l'Inde et de l'Egypte. Eh bien, il me semble que maintenant on a besoin de reprendre source philosophiquement et éthiquement à des pensées venue d'Orient, venues des Indiens d'Amérique aussi.

JS: $\quad$ En même temps, vous n'excluez pas l'Occident. Je pense, en particulier, à la mythologie grecque. Surtout à deux personnages que vous reprenez assez souvent: Daphné et Orphée. Est-ce que vous pouvez me parler du rôle de ces deux personnages mythologiques dans votre poésie?

AS: J'adore le baroque, les gravures du seizième siècle. J'ai vu des gravures qui représentaient la transformation de Daphné en laurier. Cela m'a plu, et j'ai commencé à écrire là-dessus. Cette idée de Daphné se transformant volontairement pour fuir la poursuite, le chasseur, encore une fois... J'adore ces rêveries de métamorphose. Orphée offre aussi une métamorphose. Au profit de quoi? Du chant. Tout poète aime Orphée, le rapport Orphée-Eurydice. Je vais souvent en Grèce, dans les îles grecques. L'été, j'y suis souvent allée.

JS: Vous l'évoquez dans Calendrier solaire.

AS: Oui. Calendrier solaire, ce ne sont que des textes sur la Grèce, à travers les années. Bien sûr, je ne renie pas l'Occident. Je suis complètement occidentale. Mais je pense que l'avenir, que je vois avec couleurs un peu utopiques, est dans le mélange des êtres, des 
cultures.

JS: Dans Calendrier solaire pourquoi est-ce que vous avez choisi de vous exprimer par le poème en prose ou la prose poétique, plutôt que le poème en vers? Quand et pourquoi choisissez-vous d'écrire des poèmes en prose?

AS: Là, il y a quelque chose de très contingent. Ce Calendrier solaire est le produit de mes notes de voyage - Une fois en Chine, j'ai écrit des textes sur la Chine qui ont paru et sont en prose. En Grèce aussi j'écrivais, je prenais des notes sur mes émotions. Puis, peu à peu retravaillant, j'ai dû passer, de la prose poétique au poème en prose. Je crois que dans ce livre, s'il y a un défaut, c'est cela, je n'en sais rien, d'alterner la prose poétique et le poème en prose. Je sais qu'il y a des textes qui sont des poèmes en prose parce que, d'emblée, ils se proposent quelque chose qui est de l'ordre de la poésie, du poème. D'autres se sont faits plus discursifs. Ce rythme ample du poème en prose me paraissait couvrir le sujet même. La Grèce, les îles grecques, la mer, ce n'est qu'ampleurs et lignes horizontales.

JS: À ce moment-là, faire des poèmes en prose dans ce cas, c'était marier forme et fond en quelque sorte?

AS: $\quad$ Il me semble que oui, il y avait une adéquation. Mais il y en a un qui porte le titre "Calendrier solaire", qui est venu plus en poème. Je me laisse guider par les rythmes, comme tout poète. Et quand un rythme ne s'impose pas, un rythme de vers, je n'en ai pas le désir, j'écris sans aller à la ligne. Avec un désir d'authenticité, aussi. De ne pas faire quelque chose de gratuit. De ne pas couper gratuitement. Quand je ne suis pas sûre d'avoir à couper, je ne coupe pas.

JS: $\quad$ Il me semble qu'une des autres sources d'inspiration de votre poésie, c'est l'amour de vos deux fils, Manolo et Jérôme. Vous avez écrit plusieurs poèmes à vos deux fils, en particulier un recueil qui s'appelle Le Poème de mes fils. Ce sont des poèmes très beaux, très tendres. Pouvez-vous parler des liens que vous ressentez entre l'amour pour vos deux fils et l'élan vers la poésie?

AS: J'ai rassemblé dans ce recueil Le Poème de mes fils, des poèmes faits au cours du temps à mes fils, à mes enfants. Ils m'en ont inspiré quand ils étaient petits, entre quatre ou cinq ans. Après, lorsqu'ils étaient adolescents (période difficile), ils étaient beaux comme les adolescents garçons le sont quelque fois. J'en ai fait d'autres sur eux après, plus grands. Oui, j'ai trouvé intéressant de les rassembler parce que cela représente une évolution n'a pas tellement été fait, je crois.

JS: Justement, il y a très peu de mères qui ont fait des poèmes sur leurs enfants. 
AS: $\quad \mathrm{J}^{\prime}$ allais dire bêtement; je suis très maternelle, mais la plupart des mères sont très maternelles! Donc, c'est pas ça.

JS: Pourtant, la plupart des mères ne font pas de poèmes!

AS: C'est vrai. Oui. Mais comme ils le disent parfois, ces poèmes, les enfants qu'on peut avoir, c'est la vie même. On les sent comme la vie même. Là, il y a sûrement un rapport entre l'amour et la poésie. Tout art est généreux. Même si les gens sont infects, humainement, quand ils font une oeuvre, elle est élan de générosité. Toute oeuvre, c'est se livrer, donner, donner de la vie au monde. Comme on donne la vie et l'amour à ses enfants, l'oeuvre fait plus ou moins la même chose, ne pensez-vous pas?

JS: $\quad$ Si. Je suis d'accord avec vous. Mais, à un certain niveau vous êtes quand même divisée entre cet amour, cette générosité, c'est-à-dire la poésie en tant que don que vous présentez au lecteur, et une critique sociale. Dans un poème, par exemple, vous parlez de la "Mondial Machine Society", etc. À cet égard, je pense aussi à un personnage un peu bizarre mais fascinant créé par vous qui s'appelle "Popo". Ce personnage, dans Le Désarmement intérieur, me rappelle un petit peu le personnage de Plume de Michaux. Qu'est-ce qui vous a inspirée à imaginer ce personnage satirique de Popo?

AS: D'abord, "popo", cela veut dire "poème" - ou antipoème. J'ai fait ces textes de dérision sur Popo il y a un an ou deux. Je vois assez souvent le monde sous des couleurs comiques, que mes poèmes traduisent rarement. Quand j'ai envie d'écrire, c'est plutôt sur l'émotion lyrique, finalement. Cette petite série sur Popo m'amusait. Je ne crois pas que ce soit là ma veine principale, mais ils disent autrement. Voilà. Permettez-moi de dire que je trouve que j'ai très longtemps écrit de mauvais poèmes. Dans Terra nostra je n'en ai repris que très peu des premiers. Pourquoi? Parce que j'écrivais innocemment, premier défaut. Je croyais travailler, mais je travaillais très peu mes poèmes. J'avais très peu de temps: j'avais des enfants, tout ça. Puis, je n'osais pas y passer trop de temps. Culpabilité... Ce sont des conditions objectives pour écrire de mauvais poèmes. Je ne veux pas qu'on réédite les premiers in extenso. Je trouve que cela n'a aucun intérêt.

JS: $\quad$ Il y a beaucoup de poètes qui diraient la même chose, mais c'est dommage, quand même! Moi, je dirais plutôt que vous êtes passée de poèmes beaux et simples, au début, d'une belle simplicité, à beaucoup plus de complexité dans des recueils plus récents, où il $\mathrm{y}$ a plusieurs niveaux de métaphores et où vous créez des dialogues entre plusieurs niveaux de métaphores, comme, par exemple, dans Figures du temps sur une eau courante.

AS: $\quad$ En effet. C'est vrai que dans Récit des terres à la mer, en 1978, j'ai réellement 
appris à écrire, en faisant ce travail, mettons vers 1975. Là, j'ai coupé, j'ai voulu donner une unité d'écriture, j'ai enlevé le pronom personnel "je", j'ai beaucoup malaxé. À partir de là, j'ai moins eu d'innocence et de naïveté pour écrire. J'ai un peu appris à écrire.

JS: $\quad$ Avec ce recueil c'est une nouvelle étape qui commence, oui. J'aimerais que l'on parle aussi des influences littéraires, des affinités que vous ressentez avec d'autres poètes. Je pense, tout d'abord, à André Chedid. Dans Terra nostra vous avez dédié les "Sonnets des questions" à Andrée Chedid. Je sais aussi que vous êtes de grandes amies. Pourquoi est-ce que vous vous sentez proche de Mme Chedid? De sa poésie et de sa personne?

AS: J'ai lu ses romans, d'abord, que j'ai admirés. Puis, quand je l'ai connue, j'ai vraiment admiré sa générosité, sa simplicité. C'est ça que j'aime chez les gens. Je ne peux pas supporter les artistes prétentieux. D'Andrée, j'ai aimé son humanité, son humilité, alors qu'elle fait une vraie oeuvre, une très belle oeuvre. On sent un grand courant généreux. Il n'empêche qu'il y a une grande exigence d'écriture. En fait, c'est à travers elle que nous nous connaissons, vous et moi.

JS: $\quad$ Effectivement! Oui. Et puis, quels sont les autres écrivains contemporains avec qui vous ressentez des affinités, que vous avez influencés ou qui vous ont influencée? Je sais qu'il y a plusieurs poètes lyonnais que vous connaissez très bien.

AS: Moi, je n'ai influencé personne, je crois, jusqu'à présent! Mais quand j'ai écrit mon premier livre, je venais de lire René Char. Je l'ai connu, René Char. J'ai une correspondance avec lui, une petite correspondance. Je lui ai envoyé mon premier livre et il m'a encouragée vraiment à écrire. Je crois que dans mes premiers recueils il doit y avoir une façon de mettre des mots abstraits, qui est une influence de René Char, devenue défaut chez moi! son exigence envers lui-même a beaucoup compté à mes yeux. Si je dois dire certains des poètes que j'aime, voici William Blake, Hölderlin et Novalis. Rilke surtout, Got Ritsos. Plusieurs du Sud. Ungaretti, etc. Des poètes espagnols aussi. J'aime beaucoup un contemporain que j'ai traduit, Luis Antonio de Villena. J'ai aimé Machado, Lorca. Mais toute la littérature espagnole. Je vous parlais de Manrique tout à l'heure. Je relis St. Jean de la Croix. Tous ces poètes mystiques... Et aussi la veine picaresque. J'aime beaucoup la littérature espagnole. En plus, la langue espagnole. Elle est concrète, pleine de synonymes. C'est une langue qui porte la poésie bien plus que le français - abstrait, pas rythmé.

JS: Vous avez mentionné William Blake. Justement, cela à voir avec ma prochaine question: une question sur l'art. Vous avez collaboré avec des artistes comme Jacques Clauzel. Vous avez créé de très beaux livres d'artiste ensemble. Qu'est-ce qui vous a amenée à faire ce travail? Quelle sorte de rapport entre poésie et image se montre dans ces livres? Est-ce que vous avez envie de continuer à faire ce genre de travail et pourquoi? 
AS: Au début, ça été par hasard. Par Marc Pessin, qui fait de beaux livres. J'en ai fait deux chez lui, dans la collection "Le Verbe et l'empreinte". Marc Pessin m'a demandé des textes. (Entre parenthèses, je n'ai eu aucune politique pour ma carrière. Ma seule politique, c'est de n'avoir aucune politique. À partir de là, il se trouve qu'il y a eu plusieurs livres d'artiste. Ça veut dire aucune diffusion. Ils sont repris dans Terra nostra, mais dix ou quinze ans ont passé avec très peu de publications à cause de ça). J'en ai fait rois avec Clauzel. Je vais en faire un quatrième. Après, un ami poète de Paris, Jean-Marc de Benedetti, m'a demandé des textes. Paradoxalement, dans la discrétion actuelle de la poésie, du moment qu'on me demandait un livre, je disais oui, sans songer qu'il faudrait peut-]être faire des livres un peu plus diffusés. Puis, ces livres se créant, j'ai eu grand plaisir au contact avec les artistes et à l'objet-livre, évidemment.

JS: $\quad$ Vous m'avez dit que dans la plupart de ces collaborations, il ne s'agit pas d'une illustration des poèmes, que c'est un travail séparé, différent. Cependant, dans votre prochaine collaboration avec un artiste, ce sera plutôt une illustration de vos poèmes.

AS: C'est vrai. Michel Roncerel, celui qui fabrique "Manière noire" est un très bon graveur. Il m'a demandé des textes, que je lui ai envoyés récemment. Lui, il aime partir des textes. (Roncerel/DeBenedetti, Pessin, Clouzel. Voilà, on poursuit.

JS: Vous habitez Lyon depuis longtemps. Comment est-ce que cette ville a influencé votre poésie, votre façon de voir le monde? Il s'agit d'une influence profonde, non?

AS: Oui, puisque j'y vis depuis quarante ans! C'est une ville que j'aime. Que je déteste aussi, mais enfin, bon... C'est une belle ville, quand même.

JS: Il y a, par exemple, les deux fleuves, la Saône et le Rhône.

AS: C'est ça. Effectivement, c'est très porteur de rêveries, la Saône plus ou moins féminine et le Rhône, violent. Je suis entre Saône et Rhône. C'est une ville au riche passé: les Canuts, les traditions révolutionnaires. C'est une ville neutre, une ville marchande aux traditions bourgeoises et déplaisante, froide. Où on se sent très seul, abandonné à soimême. C'est ça que je cherche, au fond, à être livrée à moi-même. Là, je vis seule, face à moi. C'est la condition de l'écriture. Souvent je pense à Thérèse d'Avila, qui, au bout de vingt ans de couvent, a écrit à peu près: "Je n'ai réellement progressé, spirituellement, que lorsque je me suis enfermée. Parce que les vingt premières années, je recevais des visites..." Sa vraie démarche mystique, elle dit qu'elle a eu lieu vingt ans après. Elle a commencé à ce moment-là réellement. Eh bien, moi — révérence gardée (ça fait deux fois que je fais allusion à des mystiques espagnols!) - je pense souvent à ça, et que ma petite démarche de poète, pour dire quelque chose qui arrache les tripes, quelque chose d'authentique, ça 
s'est fait dans la solitude. Et ça, à Lyon, pour la solitude, c'est épatant! Mais je veux vous dire aussi qu'actuellement, il y a tout un groupe - vous savez, comme il y a eu au seizième siècle - un groupe de poètes une dizaine de vrais poètes: Patrick Laupin, Hervé Micolet, Béatrice de Jurquet. François Montmaneix, Roger Dextre etc. Oui, vraiment, il y a un groupe. Hétéroclite, mais il serait intéressant de voir ce qu'ils ont en commun dans leur rapport à Lyon. Sans doute une façon de couper aux modes et pour chacun de creuser son sillon dans la solitude. Ça, c'est un vrai rapport à Lyon.

JS: J'aimerais vous poser une question sur le développement récent des études critiques de la poésie féminine en France. (J'utilise le terme "poésie féminine" entre guillemets!). Pendant très longtemps la critique française et les cours à l'université en France et à l'étranger ont plus ou moins exclu les poètes femmes françaises du vingtième siècle. Maintenant, cela commence à changer. Où est-ce que vous vous situez par rapport à ce développement?

AS: Je vois bien que je fais partie des femmes qui se sont obstinées; qui ont continué à écrire. Je vois aussi je suis dans diverses Anthologies, mais pas celles de ces dernières années: les livres d'Artiste m'ont desservie provisoirement. L'actualité est pressée. Et les chercheurs comme vous rares! Il faut faire l'oeuvre; critique et université suivront. Avec retard que les messieurs poètes sont très gentils avec nous mais qu'ils ont un peu tendance à jouer, eux, dans la cour des grands. Être poète, femme et à Lyon, ce n'est pas la meilleure position de marketing! Je suppose! Parfois c'est dur. Parfois ça m'énerve. Je me dis: "Tous ces efforts, pour quoi?" puis, la plupart du temps, je n'y pense pas. J'écris, ça suit son bonhomme de chemin. Et il y a la reconnaissance de tel ici, de tel, de tel ailleurs... Je ne me pose pas trop la question de la promotion. Même je ne me la pose pas du tout.

JS: Évidemment, avec le roman, la promotion est plus facile, car les gens ont beaucoup plus l'habitude de lire des romans. Ça peut être l'évasion, la consommation. Et puis, il est très facile de s'accrocher à des "personnages" et à l'intrigue. Alors que, personnellement, je préfère la poésie parce qu'elle présente des défis de lecture bien autres que ceux que le roman (ou la plupart des romans actuels, en tout cas) pourrait présenter. Vous avez dit que la poésie c'est "la flamme qui brûle l'être".

AS: $\quad$ Lire la poésie, c'est un exercice de liberté. Lire de la prose, d'abord cela fait appel à l'intelligence les êtres humains sont intelligents. C'est une prise sur le monde et une évasion éventuellement. Une évasion, et une invasion, quand elle est riche. La poésie d'emblée demande un effort, un peu comme la musique contemporaine. Je comprends que beaucoup de gens n'aiment pas la poésie. Elle donne, la poésie, mais il faut chercher. En plus, toutes les ressources de l'écrit tendent à diminuer. On en voit les traces partout, depuis les fautes d'orthographe jusqu'à l'absence de lecture. Bon l'image est là! Elle donne aussi, 
l'image, évidemment. Il est vrai que la poésie, celle du poème, pour le moment est en mauvaise posture. Mais disons, l'être: pour moi, elle est le dire même de l'être. Elle est la parole. Les arts sont multiples, mais la poésie, nous parlons de celle du poème ici, redit la première parole de l'être humain. Elle redit l'être humain au monde, elle se répète et demeure à travers les siècles et les millénaires.

JS: La poésie pour vous, c'est surtout le chant. J'ai l'impression que vous utilisez le mot "chant" plus volontiers que le mot "poème" pour parler de votre travail.

AS: Oui, parce que je ne suis pas théoricienne. J'ai beaucoup lu de la philosophie, des essais, mais guère de théorie sur la poésie. Oui, la poésie, profondément, est chant, il me semble.

JS: Maintenant que vous avez publié Terra nostra et que vous revoyez toute votre carrière de poète, quels sentiments est-ce que vous éprouvez à la publication de ce texte?

AS: Je tiens assez à ce livre, puisqu'il reprend quelques poèmes de mes premiers livres, plus les livres d'artistes et des inédits. Il me semble qu'il montre la cohérence d'une voix. Dans le chaos d'une vie - on tâtonne dans sa propre vie - on voit des cohérences. Il montre une obstination, une persévérance dans l'être... Sans cesse, le poème reconstruit le monde qui nous détruit, il remonte le cours d'un fleuve que nous descendons, il est jaillissement, lumière, il est le Verbe, sa profération.

JS: J'ai ressenti en lisant ce livre l'importance de l'idée du partage. En effet, son titre, c'est Terra nostra, non pas Terra mea.

AS: Ah oui. Il y a plusieurs poèmes dans les inédits comme, par exemple, À la terre. Cela rejoint ce qu'on disait précédemment, la prise de conscience d'une appartenance que nous faisons tous laborieusement, qui me paraît si nécessaire. Je me sens élément de la terre, comme faisant partie de la terre. On sait que l'éclatement d'étoiles a fait la vie et donc, la terre. J'avais envie de dire cela l'unité. Cette prise de conscience du fait que nous marchons tous du même pas, aussi. J'ai ressenti souvent que lorsqu'on torturait quelque part, c'était moi qui torturait et était torturée.

JS: Je me souviens d'un de vos poèmes des années $60 / 70$, où la poète se réveille au milieu de la nuit et elle voit des scènes de torture dans le monde entier. Et à la fin du poème, elle entend le cri d'un enfant. Donc, il y avait là à la fois cette vision vaste de la violence et de la torture à l'échelle mondiale, et une prise de conscience par rapport à cela, et, en même temps, du côté personnel, une reconnaissance de la place des enfants dans la vie humaine à travers l'acte de rassurer l'enfant, dans cette nuit de l'être. 
AS: Quand j'ai eu mes enfants, j'étais obsédée par l'idée qu'ils allaient pouvoir partir à la guerre, être torturés. Après la Guerre d'Algérie, que j'ai suivie, j'ai longtemps pensé à cette question de la torture, par rapport à nos enfants.

JS: Est-ce qu'une des raisons pour lesquelles vous écrivez de la poésie, c'est pour dénoncer la violence?

AS: C'est-à-dire, je trouve qu'on se remet mal du malheur d'être au monde dès qu'on apprend qu'il y a les guerres, la violence. Quand j'ai appris qu'il y avait des riches et des pauvres, ça m'est tombé dessus. "Quoi? Mais comment? L'humanité a attendu jusqu'à moi pour régler ça? Quand je serai grande, j'arrangerai ça!" Donc, comme dit Sartre, toute vie est l'histoire d'un échec! (Rires) Ces malheurs dans lesquels on trempe alimentent la poésie. Ça alimente le désir de chant, qui est l'autre versant, celui de la gazelle.

JS: Je sais que vos origines occitanes font partie de votre vie de poète et de femme. Par exemple, vous avez écrit un roman historique, Marie de Montpellier, où vous puisez dans l'histoire de cette région. J'ai remarqué en lisant votre poésie un poème où vous citez au début un vers de la trobairitz la Comtesse de Die, qui a écrit: "Je chanterai ce que je n'aurais pas voulu chanter," Dans ce poème, vous faites référence à la tradition des troubadours. Qu'est-ce que cette tradition représente pour vous en tant que poète?

AS: D'abord, "Je chanterai ce que je n'aurais pas voulu chanter": je savais déjà en mettant cette citation que ça parlait aussi de moi. Je n'aurais pas voulu être poète. Je n'aurais pas voulu avoir une vie compliquée. J'aurais aimé avoir une vie simple. Écrire c'est aller contre soi: "je chanterai le malheur que je n'aurais pas voulu chanter", "je chanterai la difficulté que je n'aurai pas voulu chanter". Ceci dit, justement, les troubadours. Pour vous dire à ce propos deux mots de mon père, qui venait de Montpellier, il a toujours chanté, toute sa vie. Il y avait même un jeu où il me parlait et je lui répondais en vers; on faisait des poèmes. (Ça n'allait pas très loin...). Il me semble qu'il y a des traditions - un goût populaire du bien parler, des jeux de mots, de la poésie. Par ailleurs, les troubadours, c'est l'expression du Sud. Effectivement, j'ai aimé lire les troubadours. Je me plais à cette rêverie des poètes du douzième siècle, à ce raffinement.

JS: De la fin amors, l'amour de loin? La plainte du chevalier qui recherche la dame inaccessible et qui chante son malheur de ne pas être avec elle?

AS: Je crois que "l'amour de loin", ça m'inspire assez. Comme s'il y avait une dichotomie profonde et jamais dominée entre l'amour physique et un amour conceptuel. 
JS: Quels sont les projets que vous poursuivez et que vous pensez mener à bien dans les années qui viennent?

AS: Pour l'instant, j'écris une espèce de biographie d'enfance. C'est une oeuvre en prose. J'ai aussi le désir d'écrire d'autres poèmes; j'en écris par ci, par là. Mais pour l'instant mon projet le premier, c'est ce livre, qui s'appellera La Cage à grillons ou Refus d'obéissance. Quand j'étais petite, il y avait à la campagne de petites cages où je retenais un grillon avec une feuille de salade et il chantait. J'ai des journaux de voyages que j'aimerais mener à bien, et de la poésie encore, sous toutes formes.

JS: Merci beaucoup, Annie!

\section{Notes}

${ }^{1}$ Lyon, le 20 juin 1998.

${ }^{2}$ Choix de publications. Poésie: La nuit introuvable (Henneuse, 1961); La femme-buisson (Saint-Germain-des-Prés, 1973); Les fous de Bassan (Saint-Germain-des-Prés, 1976); Récit des terres à la mer (Federop, 1978); Figures du temps sur une eau courante (Belfond, 1983); Les dieux manquent de tout (Paroles d'Aube, 1996); Calendrier solaire, gravures Marc Pessin (Le Verbe et 1'Empreinte, 1997); Le poème de mes fils (Ed. En forêt, 1997); Terra nostra (Le Cherche-midi, 1998). Récit: Marie de Montpellier (Presses du Languedoc, 1991). 